PROTEOSTASIS in health and diseases

\title{
Book Chapter X \\ Title: Resistance to the proteasome inhibitors: Lessons from multiple myeloma and mantle cell lymphoma
}

Authors: Maria Gonzalez-Santamarta, Grégoire Quinet, Diana Reyes-Garau, Brigitte Sola, Gaël Roué and Manuel S. Rodriguez.

\begin{abstract}
Since its introduction in the clinics in early 2000s, the proteasome inhibitor bortezomib (BTZ) significantly improved the prognosis of patients with multiple myeloma (MM) and mantle cell lymphoma (MCL), two of the most challenging B-cell malignancies in western countries. However, relapses following BTZ therapy are frequent, while primary resistance to this agent remains a major limitation for further development of its therapeutic potential. In the present chapter, we recapitulate the molecular mechanisms associated with intrinsic and acquired resistance to $\mathrm{BTZ}$ learning from $\mathrm{MM}$ and $\mathrm{MCL}$ experience, including mutations of crucial genes and activation of pro-survival signalling pathways inherent to malignant $B$ cells. We also outline the preclinical and clinical evaluations of some potential druggable targets associated to BTZ resistance, considering the most meaningful findings of the past 10 years. Although our understanding of BTZ resistance is far from being completed, recent discoveries are contributing to develop new approaches to treat relapsed $\mathrm{MM}$ and $\mathrm{MCL}$ patients.
\end{abstract}

\section{Keywords}

BTZ resistance, proteasome, ubiquitin, mantle cell lymphoma, multiple myeloma.

\section{Introduction}

Proteolysis is tightly regulated in eukaryotes through the superposition of sophisticated molecular mechanisms to ensure protein homeostasis. One of the major proteolytic activities is driven by the $26 \mathrm{~S}$ proteasome that holds a catalytic core particle (CP) or 20S [1]. The proteolytic activity of the $26 \mathrm{~S}$ proteasome requires the previous ubiquitylation of protein targets mediated by a cascade of thiol-ester reactions implicating at least 3 enzymes named activating (E1), conjugating (E2) and ubiquitin ligases (E3). Removal or remodelling of ubiquitin chains conditions the stability, localization and function of the modified target proteins. The ubiquitin tagging step and the $26 \mathrm{~S}$ mediated-proteolysis constitutes the Ubiquitin Proteasome System (UPS). Some proteins directly degraded by the $\mathrm{CP}$ do not require ubiquitin tagging and are therefore destroyed by an ubiquitinindependent process. The CP can also include proteasome subunits that are specifically involved in the immune response, constituting the immunoproteasome. Furthermore, the $\mathrm{CP}$ can be associated to other regulatory subunits such as $11 \mathrm{~S}$, which have specialized cellular functions [1]. In sum, the proteasome acts as a central hub of cellular proteolysis, having an impact on multiple processes such as cell cycle, DNA repair, cell differentiation, immune response, amino acid recycling or apoptosis. For this reason, the proteasome became a privileged target for drug development to treat diverse disorders including cancers, infections and inflammation-related diseases, among others [2]. The proteasome 
inhibitor (PI) bortezomib (BTZ), also known as Velcade, was, in 2003 and 2006, the first Food and Drug Administration (FDA)-approved PI for the treatment of two haematological malignancies: multiple myeloma (MM) and chemotherapy-resistant mantle cell lymphoma $(\mathrm{MCL})$, respectively. Despite the success of BTZ therapy, inherent and acquired resistance in patients were observed, encouraging the development of a new generation of Pls, as well as small molecules targeting enzymes of the UPS. Full understanding of the mechanisms underlying BTZ resistance in cancer is a prerequisite to design new strategies to recover sensitivity to these agents, or to use alternative treatments to lower apoptosis threshold in BTZ-resistant cells. To elucidate these mechanisms several laboratories have characterized a number of patient-derived MM and MCL cell lines with natural or acquired resistance to BTZ. In this chapter we summarize mechanisms of PI resistance reported in the last decade. Even if some of these acquired resistance mechanisms have not yet been confirmed in patients, their discovery may have an impact in upcoming clinical studies. We also review potential strategies to overcome PIs resistance mechanisms, including the use of new signalling pathways inhibitors regulating protein homeostasis.

\section{Cancers treated with proteasome inhibitors}

Resistance to proteasome inhibitors has been observed in various cancer types including haematological, pancreatic or breast cancer [3]. Two of the best responding cancers are $\mathrm{MM}$ and $\mathrm{MCL}$ and for this reason, more knowledge has been accumulated on BTZ resistance for these hematologic disorders [4].

\subsection{Multiple myeloma}

$\mathrm{MM}$ is a plasma cell malignancy with bone marrow (BM) infiltration of clonal cells and monoclonal immunoglobulin protein in the serum and/or urine of patients. Genomic techniques have allowed a better understanding of the genetic abnormalities of $\mathrm{MM}$ by providing a better landscape of this collection of diseases with a common clinical phenotype [5]. Several genetic alterations including chromosomal translocations of the immunoglobulin heavy chain (IGH) gene leading to the overexpression of D-type cyclin, have been considered as primary events. Not less important are the secondary mutations and clonal evolution. The most frequent mutations occur in KRAS, NRAS, FAM46C, DIS3 and TP53, among others. These mutations affect multiple signalling pathways by altering the mRNA levels but also protein expression and stability. In the past decade, this knowledge has contributed to remarkable changes in the clinical practices, such as the implementation of more effective therapies including new classes of drugs like PIs. The combination of BTZ with immunomodulatory drugs (IMiDs) such as lenalidomide or dexamethasone are currently among the most effective treatments in MM (see section 4). The success of BTZ as a MM treatment underlies its broad impact on the stability and activity of vital cellular factors.

\subsection{Mantle cell lymphoma}

$M C L$ is an aggressive non-Hodgkin lymphoma ( $N H L$ ) arising from pre-germinal centre of mature $B$ cells and is typically incurable due to the inevitable development of drug resistance, leading to the worst prognosis among NHL subtypes [6]. Classical MCL cells show minimal mutations in the IGH variable region gene (IGHV) and express the transcription 
factor SOX11. Patients present tumours in lymph nodes or extra-nodal sites and cells overexpressing cyclin D1 prone to acquisition of additional abnormalities in cell cycle, DNA damage response, or cell survival pathways, leading to a more aggressive disease behaviour. Less typical are leukemic non-nodal MCL developed through the germinal centre with IGHV somatic hypermutation and minimal SOX11 expression. These patients present MCL cells in peripheral blood, BM and spleen. Leukemic non-nodal $\mathrm{MCL}$ behaves in a more indolent way with genetic stability over time. Secondary genetic abnormalities such as TP53 mutations, result in a more aggressive disease associated with poor outcome. Since BTZ approval by the FDA in 2006 for the treatment of relapsed/refractory (R/R) MCL, numerous phenomena have been described to explain innate or acquired resistance observed in more than half of patients [7]. It is known that the development of resistance to BTZ in $\mathrm{MCL}$ is an adaptive process, which takes place gradually and includes metabolic changes and/or deregulated (re)activation of adaptive processes like plasmacytic differentiation, autophagy, or improper activation of intracellular signalling pathways such as PI3K/AKT/mTOR axis or NF-KB, among others.

\subsection{Proteasomes and chemical inhibitors}

The proteasomes are macromolecular proteolytic complexes with distinct roles under multiple physiologic or pathologic situations. The $26 \mathrm{~S}$ proteasome is composed by a $19 \mathrm{~S}$ regulatory particle that recognizes ubiquitin chain as degradations signals [1]. The catalytic core or 20 subunit contains $7 \alpha$ and $7 \beta$ subunits of which $\beta 5, \beta 2$ and $\beta 1$ hold the chymotrypsin-like, trypsin-like and peptidyl-glutamyl peptide-hydrolysing activity. Alternative $\beta$ subunits named $\beta 5 i, \beta 2 i$ and $\beta 1 i$, are expressed in haematopoietic cells in response to pro-inflammatory signals such as cytokines or $\gamma$ interferon and integrate the immunoproteasome. The $20 \mathrm{~S}$ core can also associate with $11 \mathrm{~S}$, another regulatory particle also known as PA28, REG or PA26 which contributes to the action of the immunoproteasome but can also drive proteolysis in other cellular compartments such as the nucleus [1]. Given the role of the proteasome in the degradation of many critical cellular factors, its potential as therapeutic target attracted the interest on many pharmaceutical companies.

Approved in the 2000s by the FDA, BTZ has been used for decades as one pivotal treatment in MM and MCL. However, its association with neuropathy and the acquisition of resistance in the clinics highlighted the need to develop new PIs that would be more effective, less toxic and will reduce the occurrence of resistance. Each of these aspects was considered for the development of second generation PIs such as marizomib (MRZ), carfilzomib (CFZ), ixazomib (IXZ) and oprozomib (OPZ) [3] (Table 1). Unlike BTZ, some of them target all the catalytic sites of the proteasome, like MRZ. They carry a different administration way and reversibility than BTZ, hence reducing off-target effects and toxicities in patients. MRZ and OPZ are in early clinical development, and CFZ and IXZ have been already approved in combined treatment for R/R MM. However, preclinical adaptation to these new agents has already been reported, strengthening the need for alternative strategies to face PI resistance [8]. 


\section{Molecular origin of the resistance to bortezomib}

In the last decade, several molecular mechanisms involved in BTZ resistance have been proposed. During the progression of the disease, complex genomic alterations promote the activation of different signalling cascades that contribute to the development of the resistant phenotype. These include defects in the initiation/regulation of cellular stress, cell differentiation, apoptosis and autophagy, in combination with mutations and alterations in the expression of the drug target. On the other hand, microenvironmental factors and epigenetics can be another source of inherent resistance mechanism, as these events can modulate the expression of critical genes, including tumour suppressors [9]. The acquired resistance to BTZ is also multifactorial including, among others alterations in the levels of expression of proteasome subunits, crosstalk with other proteolytic pathways or overexpression of efflux pumps.

\subsection{Inherent resistance}

In this part of the chapter, we will review available data about molecular mechanisms that have been proposed so far to be at the origin of the inherent BTZ resistant phenotype in $\mathrm{MM}$ and $\mathrm{MCL}$.

\subsubsection{Mutations in PSMB5 proteasome subunit}

PSMB5 mutations are known to lower PI binding capacity and to impair the chymotrypsin-like catalytic activity of the $20 \mathrm{~S}$ proteasome $[10,11]$ giving a benefit under $\mathrm{PI}$ stress. However, both in in vitro and in vitro settings, mutations were detected only in tumour cells having received heavy PI-based therapies, suggesting that the selected mutations emerge lately during the process of clonal selection beside the apparition of the resistant phenotype. Moreover, in vivo, at the time of relapse, subclones exhibiting PSMB5 mutations could partially or totally disappear, questioning the role of such mutations at late stages of the disease [12]. Finally, the relevance of PSMB5 gene mutation in BTZ-resistance has recently been challenged. Soriano and colleagues have shown that proteasome activity is dispensable in BTZ- and CFZ-resistant MM cell lines suggesting that PSMB5 mutations are likewise not required or involved in the development of BTZ-resistance [10].

In support, no mutations of PSMB5 were found for years in MM primary cells, even using targeted or high-throughput sequencing techniques, of large cohorts of patients including refractory patients or in relapse. The relevance of PSMB5 mutations and their functional impact were suggested recently. Four PSMB5 mutations were detected in a single MM patient having received prolonged BTZ-based treatments (Table 2) [12]. According to the darwinian model of myeloma evolution, mutations evolved independently in different tumour subclones. For instance, C63Y and A27P are lost during the course of the disease, whereas A20T and M45I are maintained longer. When tested in vitro, all mutations were functionally relevant and provided $\mathrm{PI}$-resistance but with different degrees according to both the mutation itself ( $A 20$ and M45 having a higher impact than $\mathrm{C} 63$ and $\mathrm{A} 27$ ), and the PI tested (BTZ, CFZ or IXZ). The response pattern was similar for BTZ and IXZ, but not for CFZ. CFZ response was less affected by PSMB5 mutations, likely due to its unique structure and 
binding [12]. No mutations of PSMB5, PSMB6 and PSMB7 were ever described in CFZadapted MM cell lines [10].

\subsubsection{Apoptosis failure}

Defective apoptosis signalling is a key oncogenic mechanism of drug resistance in haematological malignancies, mainly attributed to the deregulation of B-cell lymphoma-2 (BCL-2) family members. This family of proteins is composed by prosurvival proteins such as $B C L-2, B C L-X L, M C L-1, B C L W$ and $B F L 1 / A 1)$, as well as proapoptotic factors, represented by multidomain (BAX, BAK and BOK) and BH3-only (BIM, PUMA, NOXA, BAD, BID, BMF, BIK and HRK) proteins. Once activated upon cytotoxic or stress signals, the BH3-only proteins interact with their prosurvival counterparts, leading to the release and oligomerization of BAX and BAK, permeabilization of the mitochondrial outer membrane, and the cytosolic release of apoptogenic factors, culminating in the activation of the caspase family of proteases and ultimately, cell death [13].

In MCL cells, BTZ has been described to evoke intracellular accumulation of MCL-1, which harbours a PEST sequence at the origin of its targeting to the proteasome for its degradation. As MCL-1 can physiologically interact with and block the pro-apoptotic signalling of NOXA, which is transcriptionally activated upon cell exposure to BTZ, the increase in MCL-1 levels can counteract NOXA-mediated activation of BAK, thus delaying the onset of cell death. Therefore, blocking NOXA expression or inhibiting MCL-1 was used to modulate the response to BTZ in MCL [14] (See section 4).

Despite concomitant overexpression of several antiapoptotic proteins of the BCL-2 family, MM cells depend primarily on MCL-1 for survival as demonstrated by the use of small-molecule MCL-1 inhibitor and the knockdown of MCL-1 $[15,16]$. MM cells are tightly dependent on their microenvironment known to promote MCL-1 expression in plasma cells. For example, bone marrow stromal cells (BMSCs) provide survival signals such as interleukin6 (IL-6), vascular endothelial growth factor and insulin-like growth factor. IL-6 upregulates MCL-1 transcription and induces MCL-1 dependence [17]. Recently, it has been shown that the long non-coding RNA (IncRNA) H19 is present in the serum of MM patients and that an $\mathrm{H} 19 / \mathrm{miR}-29 \mathrm{~b}-3 \mathrm{p}$ axis promotes MCL-1 translation and BTZ resistance [18]. Thus, MCL-1 is certainly an important target for coping with $\mathrm{MM}$ drug resistance.

\subsubsection{Signalling cascades}

The NF-KB pathway is activated via canonical and non-canonical signalling mechanisms. The canonical pathway regulates inflammatory responses, immune regulation, and cell proliferation, whereas the non-canonical signalling cascade leads to B-cell maturation and lymphoid organogenesis. These pathways regulate the expression of genes involved in cell survival and tumour-promoting cytokines. Therefore, its activation has profound impact in tumorigenesis. The NF-KB pathway can be potentially targeted and is expected to have a high impact on the viability of malignant $B$ cells, due to its interplay with other crucial pathways activated during B-cell differentiation, such as B-cell receptor (BCR), 
$\mathrm{PI3K} / \mathrm{AKT} / \mathrm{mTOR}$, and toll-like receptor (TLR) signalling axes. Constitutive NF-KB activity is often present in MCL and MM. The inhibition of NF-KB is a primary mechanism to induce cell apoptosis after BTZ treatment and play a role in evading the effect of this treatment in BTZresistant phenotypes [19].

A high NF-KB activity was found in tumour cells of BTZ refractory MM patients and in in vitro models of cell adhesion-mediated drug resistance (CAM-DR), reinforcing the notion that the NF-KB pathway signals BTZ resistance $[20,21]$. In $M C L$, this constitutive NF-KB signalling and consequent lack of response to BTZ has been linked to a proteasomeindependent degradation of the intrinsic NF-KB inhibitor, IKB $\alpha$ [19]. However, a number of studies have pointed out a lack of correlation between NF-KB activity and BTZ resistance status [22].

NF-KB pathway is also regulated by casein kinase 2 (CK2). CK2 is a multifaceted serine/threonine kinase involved in several cellular processes, and is overexpressed and overactive in many blood tumours. CK2 regulates signalling cascades and molecules that are targeted by BTZ. For instance, it modulates $1 \mathrm{~KB} \alpha$ protein turnover, p53 function, AKT activation, and has a role in the control of endoplasmic reticulum (ER) stress and unfolded protein response (UPR) (see section 3.2.3). Inhibition of CK2 enhances BTZ cytotoxic effect in $\mathrm{MCL}$ cell lines by down modulating NF-KB and signal transducer and activator of transcription 3 (STAT3) signalling cascades and by potentiating the proteotoxic effects of proteasome blockade. Altogether these results suggest that levels of CK2 are involved in MCL resistance to BTZ [23].

The BCR includes a heterodimer of CD79A/B molecules, and CD19, a key co-receptor. The upregulation of those molecules have been proposed to promote $B T Z$ resistance in $\mathrm{MCL}$ cells. While BCR regulates cell survival and proliferation of $M C L$ cells, in $M M$ it has been only linked to monoclonal gammopathy of undetermined significance (MGUS), a premalignant phase of MM [24]. A human phospho-kinase array further pointed out an overexpression of phosphorylated BCR kinases LYN, LCK, and YES as well as a sustained downstream activation of PI3K/AKT/mTOR axis in BTZ-resistant cells. Among these kinases, LYN was functionally associated with the resistance phenotype, rending cells more sensitive to the SRC kinase inhibitor dasatinib and allowing to synergistic activity of the dasatinib/BTZ combination in vitro [25].

In $\mathrm{MCL}$, the redox status has also been pointed out as a crucial mediator of BTZ efficacy, as PIs lead to the generation of large amounts of reactive oxygen species (ROS), modulating at least in part the transcription of NOXA and thus contributing to the cytotoxic activity of proteasome inhibition [26]. The nuclear factor NF-E2 p45-related factor 2 (NRF2) was identified as a key regulator of this response. Indeed, while under physiological conditions it is sequestrated by Kelch-like ECH-Associated Protein 1 (KEAP1) in the cytosol, when KEAP1 is oxidized by ROS, NRF2 is released to the nucleus where it initiates the transcription of genes involved in the adaptive oxidative stress response. Upon BTZ exposure, BTZ-sensitive MCL cells display a sharp increase in the expression of NRF2 target genes, as well as genes related to protein ubiquitylation or proteasome components, while resistant tumours show minimal changes. Accordingly, an elevated expression of NRF2 target genes at the basal level, predicted a poor sensitivity to proteasome inhibition [27]. In 
line with this, a recent study has highlighted the capacity of ROS to modulate some cancer stem cells (CSCs)-like subpopulations in MCL cell lines and primary cultures and to regulate cell sensitivity to $\mathrm{BTZ}$. Authors showed that $\mathrm{O}_{2}$ - was involved in the inhibition of CSC-like cells and in the sensitization of $\mathrm{MCL}$ to $\mathrm{BTZ}$, while $\mathrm{H}_{2} \mathrm{O}_{2}$ favoured a CSC-like phenotype, impairing BTZ-induced cell death [28]. This process was associated with transcriptional regulation of two $\mathrm{O}_{2}$ - and $\mathrm{H}_{2} \mathrm{O}_{2}$ targets, namely MCL-1, and ZEB-1, a WNT-regulated transcription factor that interfered with $\mathrm{MCL}$ response to chemotherapeutics. This resulted in the activation of proliferation-associated genes including $M Y C$ and CCND1 and the induction of an antiapoptotic gene signature [29].

\subsection{Acquired resistance}

$\mathrm{PI}$-acquired resistance has multi-factorial and interconnected causes and PI-resistant cells show cross-resistant profiles. Among the main mechanisms recognized in $\mathrm{MM}$ and/or $\mathrm{MCL}$ are upregulation of $20 \mathrm{~S}$ proteasome subunits including $\beta 5 \mathrm{c}$, downregulation of $19 \mathrm{~S}$ proteasome subunits and overexpression of efflux pumps. Adaptive metabolic changes, modulation of the unfolded protein response, and alteration of autophagy signalling contribute also to PI-resistance in MM cells.

\subsubsection{Overexpression of proteasome subunits}

Beside PMBS5 mutations, overexpression of PMBS5 and (to a lesser extent) PMBS6 are frequent alterations found in $\mathrm{MM}$ cell lines adapted to increased concentrations of $\mathrm{BTZ}$ $[11,30,31]$. PSMB5 is overexpressed in one MM patient with clinical resistance to BTZ, compared to three BTZ-sensitive patients [32]. Franke and colleagues demonstrated a tight relationship between impaired proteasome activity carried on by a mutated $\beta 5 c$ subunit and the $\beta 5 c$ subunit overexpression. In cells harbouring homozygous PSMB5 mutations, the upregulation of $\beta 5 c$ subunit was even more important when compared to cells harbouring heterozygous mutations. The authors propose a model in which, the prolonged exposure of MM cells to BTZ leads first to the appearance of PSMB5 mutations, resulting in decreased BTZ binding. In turn, mutant cells compensate this reduced proteasome activity by upregulation of the $\beta 5 \mathrm{c}$ subunit. Moreover, in those BTZ-resistant MM cells the upregulation of $\beta 5 c$ is associated with the downregulation of $\beta 5 i$ to balance the total proteasome units and impairs any possible remaining BTZ-inhibition [11].

In two recent studies, the involvement of the 19S subunits of the proteasome has been highlighted. To identify genes controlling the sensitivity and adaptation of MM cells to CFZ, Acosta-Alvear and colleagues used a new-generation shRNA library screening [33]. They found that the knockdown of several subunits of the $20 \mathrm{~S}$ proteasome core (including $\mathrm{\beta 5c}$ ) provides a strong sensitization to proteasome inhibition. Paradoxically, the genetic depletion of most of the $19 \mathrm{~S}$ regulatory components confers a marked resistance. They further confirmed that shRNAs-mediated knockdown of PMSC1, PMSC6, PMSD1, PMSD2, PMSD6 and PMSD12 leads to resistance towards BTZ and CFZ in MM cell lines. Importantly, the authors showed that PSMC2 levels in MM patients seem predictive for the response towards CFZ-based therapy. In the second report, Shi and colleagues used a genome-scale CRIPSRCas9 library to identify genes associated with BTZ-resistance. They validated PSMC6 depletion as the strongest hit conferring BTZ resistance in MM cells [34]. PSMC6 deficiency 
resulted in reduced $B T Z$ ability to regulate chymotrypsin-like activity of $\beta 5 c$ through changes in the proteasome structure. Mutations in other members of the PMSC group also individually impart BTZ-resistance albeit less potently. No mutation of PSMC6 has been reported so far but the analysis was performed on a cohort of untreated MM patients [34].

Recent results from our laboratories comparing BTZ-adapted MCL cell lines and their parental counterparts revealed a reduced expression of 195 proteasome subunits in BTZresistant cells. Strikingly, when autophagy was blocked with inhibitors such as bafilomycin A or chloroquine, the level of those proteasome subunits increased in resistant cells only, suggesting an autophagy-mediated degradation. In MCL cell lines that naturally resist to BTZ, the accumulation of proteasome subunits after the chemical inhibition of autophagy was proportional to the level of BTZ resistance observed. The proteasome degradation by autophagy was named proteaphagy and has been observed in response to starvation or proteasome inhibition in several biologic models including human cells, [35, 36]. Quinet et al showed that proteaphagy can contribute to develop resistance to BTZ in MCL cells since inhibited proteasomes are degraded and BTZ reduces its impact on proteasomes and cell death. In other words, BTZ-resistant cells bypass proteasome inhibition relying on autophagy through degradation of proteasomes and perhaps other cellular proteins [37].

\subsubsection{Metabolic adaptation}

Metabolic reprogramming is a hallmark of cancer that has emerged as an attractive target for novel therapeutic strategies for cancer treatment. O-GlcNAcylation is an abundant, dynamic, and nutrient-sensitive posttranslational modification that corresponds to the addition of an O-linked $\beta$-N-acetylglucosamine (O-GIcNAc) moiety to the serine or threonine residues in proteins in response to changes in the hexosamine biosynthetic pathway. As this latest depends on various essential nutrients and metabolic intermediates like glucose, glutamine, acetyl-coA, and UTP, it provides an ideal machinery for cells to sense and respond to a variety of microenvironmental conditions [38]. Little was known about its role in $\mathrm{MCL}$, until the recent study of Luanpitpong and collaborators who demonstrated that O-GIcNAcylation of tBID promoted apoptosis in MCL cells exposed to BTZ, and that this process could be amplified by co-treatment with the antifungal drug kenoconazole, an $\mathrm{O}$ GlcNAcase inhibitor that blocks tBID ubiquitylation and subsequent proteasomal degradation [39].

ABC (ATP-binding cassette) transporters such as ABCB1 (multi-drug resistance, MDR1 or P-glycoprotein, P-gp) mediate drug resistance by alterations of the absorption and elimination of xenobiotics and drugs. ABCB1 expression correlates with poor prognosis, treatment resistance and aggressiveness of the $M M$ disease [40]. $A B C B 1$ protein is overexpressed in CFZ-resistant compared to sensitive MM cell lines [10]. ABCB1 was expressed by circulating malignant plasma cells of $\mathrm{MM}$ patient at diagnosis and its expression increases along the course of CFZ-treatment [41]. Overexpressed ABCB1 protein limits the proteasome-inhibiting activity and clearance of poly-ubiquitinated proteins by CFZ and reduces its cytotoxicity. Importantly, ABCB1 overexpression affects the cytotoxic activity of epoxyketone-type PIs (CFZ) significantly stronger than non-epoxyketone PIs (BTZ). Drugs targeting $\mathrm{ABCB} 1$ may re-sensitize $\mathrm{MM}$ cells to $\mathrm{PI}$, in particular $\mathrm{CFZ}$. 
Soriano and colleagues analysed by a combined quantitative and functional proteomics approach CFZ- and BTZ-adapted MM cell lines [10]. They found that resistance to BTZ/CFZ was independent of proteasome activity but relied on energy metabolism, redox homeostasis, protein folding and degradation. PI-resistant cells adapted themselves to a very low proteasome activity while continuing to synthesize immunoglobulins. In turn, the level of metabolic intermediates involved in oxidative glycolysis (pyruvate kinase), redox state (superoxide dismutase, glutathione peroxidase, peroxiredoxin), mitochondrial respiration (cytochrome $c$ ) were increased thus maintaining high stringent redox conditions. In agreement with previous studies, authors also confirmed that the most upregulated proteins in CFZ-resistant cells were ABCB1 [40,41] and the heat-shock proteins HSP70 and HSP90, whose transcriptional regulator, HIF1, is involved in BTZ-resistance in MM cells [42] Conversely, the positive apoptosis regulators BAX, CASP and DIABLO were downregulated [10].

Similarly to Soriano et al, Dytfeld and colleagues conducted a comparative proteomic profiling of $R / R$ patients vs. naïve $M M$ patients [43]. In the proteomic signature associated with BTZ-resistance, four sets of proteins were characterized including proteasomal proteins, some factors regulating the redox status, proteins signalling apoptosis, and proteins involved in the inflammation response. In particular, regulatory and catalytic components of the proteasome, including part of the $11 \mathrm{~S}$ complex, were upregulated. The antioxidant thioredoxin, peroxiredoxin, and thioredoxin reductase were upregulated whereas annexins $\mathrm{A} 1$ and $\mathrm{A} 2$, that regulate the apoptotic process, were downregulated. $\mathrm{PI}$ resistance may thus be alleviated by manipulating the redox status and the energy metabolism.

Although not revealed by the above proteomic studies, NRF2 seems to be a node for BTZ- and CFZ-resistance in MM. As it maintains redox homeostasis by inducing antioxidant and detoxification genes and by modulating energy metabolism [44], NRF2 indirectly regulates: a) chaperoning activity [45]; b) redox, metabolic and translational reprogramming [46]; and c) activation of prosurvival autophagy. These major functions are supported by clinical data showing NRF2 upregulation in a subgroup of relapsed patients [46]. Other studies showed that high glutathione (GSH), whose levels are controlled by NRF2, dampens BTZ toxicity in MM cells [47].

In the last decade, numerous studies have indicated, that components of the BM stroma, extracellular matrix (ECM), cytokines, chemokines and growth factors, are involved in BTZ-resistance in MM cells [48] The membrane protein myristoylated alanine-rich Ckinase substrate (MARCKS), is a protein that plays an important role in cell adhesion, spreading and invasion, and is crucial for metastasis [49]. MARCKS is overexpressed in MM cell lines and is involved in the cross-resistance to the farnesyltransferase inhibitor R115777 and BTZ, as well as in MM patients that do not reach a sustained response to BTZ therapy. In addition, the inhibition of MARCKS phosphorylation, increase cytotoxicity in BTZ-resistant cells [50].

The insulin-like growth factor IGF-1, known as a growth factor for MM cells [51], is produced by plasma cells, and is present in the BM microenvironment. IGF-1 has been proposed to promote proliferation and drug resistance in MM cells through the activation of MAPK and PI3K/AKT-signalling pathways [48]. According to these data, the IGF-1/IGF-1R 
signalling axis was detected to be upregulated in three BTZ-resistant MM cell lines, compared to parental cells. Kuhn et al proved that a small molecule responsible for the inhibition of IGF-1R has the capacity to sensitize BTZ-resistant MM cells to the proteasome inhibitor [52].

\subsubsection{Protein homeostasis}

Because of their high capacity to synthesize and secrete immunoglobulins, MM cells exhibit an expanded ER network and an increased ability to cope with unfolded or misfolded proteins that accumulate in the ER. These conditions referred to as ER stress. As a consequence, MM cells activate the UPR pathway as an adaptive strategy and are rendered dependent on this mechanism for their survival [53]. ER stress upregulates three UPR signalling branches: activating transcription factor 6 (ATF6), protein kinase $R$ (PKR)-like ER kinase (PERK)-ATF4 and inositol-requiring enzyme 1 (IRE1)-X box binding protein 1 (XBP1) which suppress global translation and promote protein folding and degradation. During $E R$ stress, ATF6 translocates into the nucleus and activates the XBP1 promoter allowing an upregulation of the protein. At the same time, IRE1 oligomerizes and autophosphorylates, resulting in the activation of its endonuclease activity that cleaves XBP1 mRNA. This results in a frameshift that modifies the unspliced inactive XBP1 form (XBP1u) into an active XBP1s form. XBP1s acts as transcription factor and activates genes encoding protein folding and chaperones (see section 3.2.5). Previous studies done on cohorts of MM patients and confirmed in vitro on BTZ-adapted cell lines, defined a "low IRE1-XBP1" phenotype that predicts a poor response to $\mathrm{BTZ}[54,55]$. Moreover, XBP1 knockdown experiments in MM cell lines showed correlation with BTZ resistance, as the suppression of XBP1 lowers both the basal ER stress and the ER stress due to proteasome inhibition [55].

High expression of deubiquitinating enzymes (DUBs) and autophagy related-proteins has been detected in MM patients resistant to BTZ. These alterations in enzymes that are involved in deubiquitinating misfolded/unfolded proteins and in the turnover of proteins by the autophagy-lysosome system (ALS), suggest an important role of ubiquitin signalling pathways in BTZ resistance. Niewerth et al, shown that inhibition of USP14 and UCHL5 promotes apoptosis and helps to overcome BTZ resistance in MM patients [4]. Another enzyme that can be regulated to recover sensitivity to BTZ is the ubiquitin-conjugating enzyme $\mathrm{H} 10$ ( $\mathrm{UbcH} 10)$. Wang et al proved that through the expression of hsa-miR-631, the negatively regulation of $\mathrm{UbcH} 10$ transcription prevents $\mathrm{MM}$ cells to develop resistance against proteasome inhibitors [56].

\subsubsection{UPS-ALS crosstalk}

Eukaryotic cells have two interconnected mechanisms for protein degradation and removal of misfolded proteins and aggregates, the UPS and the macroautophagy (here referred to as ALS). Autophagy functions by double-membrane vesicles known as autophagosomes which sequester cytosolic proteins, followed by fusion with lysosomes for degradation. Autophagy is involved in several human diseases, such as neurodegenerative diseases and cancer [57]. While it appears to be tumour-suppressive in normal cellular homeostasis, autophagy can mediate tumour cell survival under stress conditions [58]. For 
many years, UPS and ALS pathways were thought to function independently, but the recent observation that impairment of either pathway impacts the other has suggested that these two proteolytic systems do collaborate [57]. It is thought that upon proteasome inhibition, autophagy is initiated as a survival mechanism to eliminate UPS substrates [59], thus upregulated autophagy could play a role in BTZ-resistance [60].

The ubiquitin-binding cargo autophagy receptor sequestrosome 1 (SQSTM1) or p62 is a critical link between UPS and ALS [61]. In CFZ-adapted MM cells, SQSTM1/p62 is elevated triggering a prosurvival autophagy however through two different mechanisms according to the settings. In the first model, the pluripotency-associated transcription factor Kruppel-like factor 4 (KLF4), is overexpressed and contributes to CFZ resistance by activating the SQSTM1 gene [46]. In the second model, elevated levels of SQSTM1/p62 conduct CFZ resistance through both a prosurvival autophagy involving GABARAPL1 upregulation and the activation of the NRF2 pathway [46]. However, since KLF4 is also a target of NRF2, both factors could cooperate for maintaining a high level of SQSTM1 transcription. The activation of NRF2 occurs through the activation of the PERK-eukaryotic translation initiation factor-2 $\alpha$ (elF2 $\alpha$ ) axis of the UPR [46]. As stated before (see paragraph 3.2.2), NRF2 is a major actor for PIresistance through the reprogramming of metabolism and the control of redox status [10, 46]. In addition to elF2 $\alpha$, another NRF2 target and translation initiation factor, elF4E3 is overexpressed in CFZ-resistant cells, and increased EIF4E3 expression was found in a subgroup of patients with chemoresistant minimal residual disease and in $R / R$ patients [46].

Interestingly, deficiency in BIM has been shown to contribute to adaptive resistance to BTZ in MM cells, mediated by increased autophagy, and that autophagy disruption by means of chloroquine could sensitize these cells to BIM-mediated cell death [62]. Of special interest, upon exposure to pharmacological inhibitors of autophagy like chloroquine or bafilomycin A, BTZ-resistant MCL cells can undergo a blockade of proteaphagy, leading to the stabilization of proteasome subunits, and the recovery of BTZ sensitivity [37]. Importantly, sensitivity to autophagy inhibitors requires a significant degree of BTZ resistance, thus suggesting that, modulating proteaphagy with specific inhibitors may be considered as a strategy to resensitize resistant cells to $\mathrm{PI}$.

\subsubsection{Stress signals}

Subsequent studies have been focused to determine the interplay between the deregulation of the intracellular stress machinery and MCL loss of sensitivity to BTZ. A key defect in BTZ-dependent cell death was first identified within the ER stress pathway, because its activation in MCL cells exposed to BTZ is required to elicit NOXA transcription [63]. ER homeostasis is controlled by the immunoglobulin heavy chain binding protein (BiP), also referred as 78-kDa glucose-regulated protein (Grp78). BiP/Grp78 forms a large multiprotein complex with a set of other ER molecular chaperones, including the Hsp90 ER homolog, Grp94, protein disulfide isomerase, calcium binding protein, and cyclophilin B [64] Under non-stressed conditions, BiP/Grp78 binds to and maintains in an inactive monomeric state the ER transmembrane PKR-like ER kinase, IRE1, and ATF6 [65]. After proteasome inhibition, the accumulation of polyubiquitylated and misfolded proteins within the ER lumen leads to BiP/Grp78 dissociation from the luminal domains of these sensor proteins and the initiation of UPR (see section 3.2.3) [66]. This coordinated cellular response initially 
promotes cell survival, but ultimately triggers apoptosis if cytoprotective mechanisms are overwhelmed. Supporting the observation that accumulation of some HSP proteins can promote cellular resistance to PIs, a correlation has been made between acquired and primary resistance to $\mathrm{BTZ}$ in $\mathrm{MCL}$, and intracellular accumulation of BiP/Grp78 in proteasome-compromised MCL cells [67]. For this reason inhibitors of HSP90 have been used to improve the BTZ-mediated cell death induction in BZT-resistant cells (see section 4) [67].

In line with this study, deregulated expression of several cytosolic HSP70 family members has also been associated with BTZ resistance in MCL [68] and MM [69]. Supporting a role of HSP70 protein in MCL cell resistance to chemotherapeutic agents, B cells modified to overexpress cyclin D1, the genomic hallmark of $M C L$, presented strong alterations in their response to growth factor withdrawal [70]. Acquired BTZ resistance is also attributed to the upregulation of other HSPs such as HSP90 and HSP27, that promote NF-KB activity [20].

\subsubsection{B-cell differentiation program}

It has been proposed that $\mathrm{MM}$ cells also achieve BTZ-resistance via the dedifferentiation of plasma cells. A pool of XBP1 $1^{\text {low/- }}$ tumour progenitors or CSCs pre-exists drug treatment, contributing to tumour diversity [54]. CSCs recapitulate maturation stages between B cells and plasma cells. Tumour B cells and pre-plasmablasts survive PI-treatment preventing cure, while maturation arrest of $\mathrm{MM}$ before the plasmablast stage enables progressive disease on PI treatment. These tumour progenitors should be targeted to allow a complete cure for MM patients.

Although MCL was originally considered a neoplasm of naive lymphocytes that have not passed through the germinal centre (GC), a significant number of cases present somatic mutations in the immunoglobulin genes, suggesting that they have been in contact with the antigen in the GC. Some cases were also described, that presented evidences of plasmacytic differentiation in patients harbouring the characteristic $t(11 ; 14)$ translocation [71]. A couple of studies have related the resistance to BTZ with the plasmacytic differentiation program in MCL cells. Plasma cells are the final effectors of humoral immunity, which are devoted to the synthesis and secretion of immunoglobulins. BTZ-resistant MCL cells display some of the characteristics of the plasma cells, such as the over-expression of interferon regulatory factor 4 (IRF4) and elevated membrane levels of CD38 and CD138 cell surface markers, but they do not present splicing of XBP1 or increase in immunoglobulin production [6]. It is postulated that during the acquisition of resistance to BTZ, the balance between the protein load and the proteasomal activity is key. When plasmacytic differentiation is induced through stimulation of TLR9 receptor, the sensitivity to BTZ changed throughout the process of B-cell differentiation to final plasma B cell phenotype with the capacity to manage the future increase in protein loads. Since the cells do not acquire full secretory capacities, this mechanism granted them with an advantage against the anti-tumour activity of BTZ. Once $M C L$ have completed this process of differentiation, they return to a BTZ-sensitive state [6]. Mouse xenograft models of MCL using different cell lines with different sensitivity to BTZ, including cells with acquired or primary resistance to the $\mathrm{PI}$, demonstrated a tight correlation between increased tumorigenicity of BTZ-resistant tumours with a plasmacytic differentiation phenotype including upregulation of IRF4, PR domain zinc finger protein 1 
(PRDM1/BLIMP-1) and CD38, and loss of the B cell markers PAX5 and CD19 [72]. Of note, beside its role as a transcription factor that represses the expression of proteins needed for B-cell identity and proliferation, and that helps to drive B cells through their final differentiation stage to become antibody-secreting cells, BLIMP-1 is also a mediator of NOXA-induced apoptosis in MCL and is required for BTZ-induced apoptosis in MCL cell lines and primary tumour samples [73]. This finding further strengthens the interplay between NOXA and plasmacytic differentiation in BTZ-mediated anticancer activity in MCL.

\section{Potential targets to recover proteasome inhibitors sensitivity}

Given the resistance to PIs observed in some patients, alternative drugs have been tested to overcome resistance as single or combinatorial treatments. Many agents have shown promising preclinical results in terms of safety, specificity and efficacy to treat PIresistant $\mathrm{MM}$ and $\mathrm{MCL}$ cells. Already existent or new therapeutic drugs are used to tackle the adaptation of the cells to PIs (Table 3). Cellular mechanisms involved in PI resistance are hence targeted by a wide range of drugs, some of the most relevant are described below.

\subsection{Deubiquitinases}

Because proteasome degradation and UPR implicate ubiquitin signal, targeting factors regulating ubiquitin signal could potentially contribute to increase the sensitivity to BTZ and therefore be an option to overcome PI resistance. In this context, DUBs have been considered as therapeutic targets to overcome BTZ resistance. P5091 is a selective inhibitor of USP7, a DUB that targets the E3 ligase HDM2. Treatment of MM cell lines and primary cells from MM patient with P5091 inhibits growth and induces apoptosis in tumour cells including those resistant to BTZ, without affecting the viability of normal PBMCs [74]. The $19 S$ regulatory particle inhibitor b-AP15 selectively blocks deubiquitinating activity of USP14 and UCHL5 without inhibiting proteasome activity. This leads to an activated UPR and an inhibited tumour growth, in MM xenografts resistant to BTZ [75]. The antitumoral effects of b-AP15 were also demonstrated in MCL [76]. Another USP inhibitor, SJB3-019A tackling USP1 showed synergic toxicity in MM when combined with BTZ [77]. Song et al found contribution of RPN11, a proteasomal deubiquitinase, in MM pathogenesis using gene expression analysis. Pharmacological inhibition of RPN11 with O-phenanthroline (OPA) or capzimin blocks proteasome function, induces apoptosis in MM cells and overcomes resistance to BTZ [78].

USP9X is also highly expressed in MM patients and in particular in those with as short progression-free survival. The partially selective USP9X inhibitor WP1130 induces apoptosis through the downregulation of MCL-1. However, this effect is transient due to the compensatory upregulation of USP24 who sustains MM cell survival. By contrast, a novel compound EOAI3402143 with a dual USP9X/USP24 inhibiting activity display promising antimyeloma activity [79]. Associated with the inhibition of USP14 activity, VLX1570 lead to an extended survival of xenografts models of myeloma including BTZ-resistant cells [80]. 


\subsection{Transport modulators}

$\mathrm{PI}$ cellular intake is regulated by transport modulators, and treatment efficiency is directly linked to intracellular concentration of drug. Specific inhibitors of ABCB1 such as verapamil and reserpine showed increased proteotoxic stress in CFZ-resistant MM cells [10]. The two HIV inhibitors nelfinavir and lopinavir counteract ABCB1 overexpression in CFZ-resistant $\mathrm{MM}$, via the modulation of the mitochondria transition pore. This promising preclinical results encourage the clinical evaluation of both treatments [41]. Thanks to its capacity to modulate the UPR pathway, nelfinavir has also attested a safe and promising activity in combination with BTZ and/or dexamethasone, in a phase I clinical trial involving advanced BTZ-refractory MM patients [81].

\subsection{Autophagy signalling}

Ubiquitin and ubiquitin-like molecules play an important role on the regulation of autophagy. In BTZ-resistant MCL cells, the use of autophagy inhibitors such as bafilomycin A and chloroquine, and the p62 inhibitor verteporfin revealed increased cytotoxicity and synergistic activity with BTZ, mediated by the reversion of the proteaphagic process [37]. Blocking autophagy also leads to multiple changes in the cell such as the accumulation of IKB $\alpha$, which prevents BTZ-induced NF-KB activation. The combination of bafilomycin A with BTZ might therefore contribute to increase cytotoxicity in MM cells in this manner [82]. Finally, orlistat, a fatty acid synthase inhibitor that affects autophagy, sensitizes MCL cells to BTZ through the inhibition of the autophagic degradation of NOXA [83].

\subsection{Oncogenes and signalling pathways}

Proteasome inhibition has pleiotropic effect within the cells. It affects a wide range of cellular factors, including important signalling cascades, oncogenes or epigenetic regulators. This modulation of critical factors during BTZ treatment can hamper the apoptotic effect of $\mathrm{PI}$, and leads to resistance. As described above, adaptation of signalling pathways including mTOR and NF-KB, or modulation of important oncogenes such as BCL-2 or MYC proteins, have been directly linked to BTZ-resistance in MM and MCL. Therapeutics agents targeting these factors have been proposed to overcome BTZ resistance.

\subsection{1 mTOR/AKT pathway}

Modulating mTOR/AKT, key proteins of a complex signalling cascade, have successfully reverted malignant cell adaptation to BTZ in preclinical studies. The dual PI3K and mTOR inhibitor dactoslisib (NVP-BEZ235) showed great results in MCL BTZ-resistant cells lines [84]. A new generation of mTOR inhibitors, such as temsirolimus and deforolimus have been also proposed for $M C L$ treatment but limited clinical impact was obtained $[85,86]$. The AKT inhibitor perifosine combined with $B T Z$ revealed increase cytotoxicity in $R / R M M$ patients previously treated with BTZ [87], warranting its use in BTZ-resistant cancers. 


\subsubsection{NF-kB pathway}

Since NF-KB pathway is overactivated under BTZ treatment, its modulation has been used to treat PI-resistant MM and MCL. Selinexor is a reversible inhibitor of exportin 1 (XPO1) that blocks the nuclear export of NF-KB /IKB $\alpha$ complexes leading to NF-KB pathway inactivation [88]. Selinexor associated with BTZ or CFZ overcomes acquired PI resistance in MM models and patients. MM drug resistance to CFZ and BTZ is enhanced in hypoxic conditions [89]. In such conditions, selinexor is capable to overcome PI resistance [89]. Because Transglutaminase 2 (TG2) is a calcium-dependent enzyme, calcium blockers have been proposed to hamper high NF-KB expression in BTZ-resistant cells. The combination of such molecules with BTZ indeed improves cytotoxicity in MCL [90]. Degrasyn has been also proposed to target constitutive NF-KB and STAT3, and combined treatment with BTZ showed a synergic apoptosis in MCL [91].

Within the BCR pathway, Bruton's tyrosine kinase (BTK) inhibitors lead to NF-KB inactivation and downregulation of MYC. Ibrutinib (Imbruvica), a first-in class BTK inhibitor, was approved by the FDA in 2013 as second line treatment for MCL patients [92]. This promising drug leads to the best complete remission rate as a single agents when compared to the other three drugs licensed at that time for use in MCL (BTZ, temsirolimus (Torisel) and the IMiD drug, thalidomide-derivative lenalidomide (Revlimid)) [93]. Promising preclinical results were obtained combining ibrutinib and BTZ in MCL and MM BTZ-resistant cells [94]. Ibrutinib alone or in combination with dexamethasone went very recently through a phase II trial with R/R MM patients, with some positive results [95].

\subsubsection{NOXA/BCL-2 proteins}

BH3 mimetic compounds like obatoclax showed great results alone or in combination with $\mathrm{BTZ}$ in relapsed $\mathrm{MCL}$. By neutralizing BTZ-induced MCL-1 accumulation, obatoclax sensitizes MCL cells to low doses of the PI [14]. A phase I/II study substantiated the tolerance of a combined treatment BTZ/obatoclax in patient with R/R MCL. However the synergism supported by the preclinical studies was not confirmed in patients [96]. Treatment of MM cell lines including BTZ-resistant cells and primary cells with cyclic adenosine monophosphate (CAMP) induces down regulation of MCL-1 and degradation of cyclin D1. Moreover, a synergy between BTZ and CAMP showed promising results in a murine xenograft model, warranting this strategy to overcome BTZ resistance [97].

\subsubsection{IRF4/MYC signalling}

As described before, exacerbated de novo IRF4 signalling has been associated with $M C L$ resistance to $B T Z$ in vitro and in vivo, thus supporting the preclinical/clinical evaluation of IRF4-targeting drugs. Following first observations in MM preclinical models where it efficiently suppressed IRF4-expressing cells [98], lenalidomide was found to be effective in vitro and in vivo in BTZ-resistant MCL tumours harbouring high IRF4 levels, while sparing IRF4 negative cases. Lenalidomide activity relied on a functional interaction with the component of the E3 ligase complex, cereblon (CRBN), and CRBN-dependent lowering of IRF4 expression, leading to the blocking of B-cell differentiation program, as shown by increase in PAX5 and loss of CD38 and BLIMP-1. Consequently, BTZ-lenalidomide 
combination could overcome BTZ resistance [72]. Lenalidomide single agent was further validated in a phase II trial involving R/R MCL patients, including cases refractory to BTZ [99]. In contrast, in MM patients, therapy combining lenalidomide with BTZ failed in phase II trial [100]. Among the IMiD family, pomalidomide has been approved by the FDA for the treatment of R/R MM with at least two prior treatments, including BTZ. When added to BTZ or MRZ in combination with low dose dexamethasone, this agent went through successful phase I trial on heavily pre-treated, high risks relapsing MM [101, 102].

Beside these approaches, the inhibition of the IRF4 target gene, MYC, in BTZ-resistant MCL cultures have been studied either with siRNA-mediated gene knockdown or with treatment with an inhibitor of BRD4, a bromodomain and extraterminal domain (BET) protein. BET proteins mainly regulate epigenetics marks. They impact gene expression and in turn, participate in cancer pathogenesis. BET inhibitors (BETis) are very promising novel anticancer agents, and combinatory therapy with these inhibitors has been suggested. Because BETis target the NF-KB pathway, their impact on BCL-2 and c-MYC proteins among others, have been tested preclinically in $\mathrm{MCL}$ and $\mathrm{MM}$. As a proof-of-concept, inhibition of BRD4 synergistically induced cell death in vitro and in vivo when combined with lenalidomide. This confirmed that exacerbated IRF4/MYC signalling is associated with $\mathrm{MCL}$ resistance to BTZ and warranted the clinical evaluation of the IMiD-BETi combination in $\mathrm{MCL}$ cases refractory to the inhibition of proteasome [72].

Also, the BETi birabresib (OTX015) significantly synergizes with BTZ, CFZ, IXZ and IMiD to improve MM response and overcome resistance to PIs. It triggers the suppression of NFKB pathway and decrease in C-MYC signalling. The birabresib/pomalidomide combination demonstrate great results to overcome adaptive resistance in MCL [103].

Moreover, JQ1, a thieno-triazolo-1,4-diazepine has been characterized as the first indirect inhibitor c-MYC transcriptional network in MM cells [104]. Thereafter, the combination of BTZ with the JQ1 derivative, CPI203, was found to be synergistic in BTZresistant $M M$ cell lines and in a primary culture from a MM patient refractory to BTZ therapy [105]. These studies supported the clinical evaluation of the IMiD-BETis combination in MM and in MCL cases refractory to PIs. An alternative approach to directly inhibit BRD4 was to promote its degradation. This was recently achieved by the use of the protein-targeting chimeric molecule (PROTAC), ARV-825, which specifically induces BRD4 ubiquitination and degradation, granting activity and overcoming PI resistance in MM [106].

\subsection{Epigenetic modulators}

Histone deacetylase 6 (HDAC6) mechanistically links the UPS and autophagy by facilitating the transport of protein aggregates along tubulin to juxtanuclear microtubule organizing centres [107]. Aggregated ubiquitinated proteins are transferred to lysosomes via autophagy, and BTZ treatment contributes to aggresome formation. Cells that lack HDAC6 were found to be defective in the removal of protein aggregates and are not able to form large aggresomes. The combination HDAC inhibitors with BTZ sensitizes MM resistant cells to proteasome inhibition [108]. Ricolinostat (ACY-1215) is a specific inhibitor of HDAC6. When ricolinostat is combined with BTZ and dexamethasone, the response rate among BTZrefractory $\mathrm{MM}$ patients raised up to $20 \%$. This combined treatment appeared well tolerated, 
supporting the use of HDAC6 inhibitors to overcome PI resistance in patient [109]. In MCL, combined $\mathrm{PI}$ and ricolinostat treatments are still under preclinical investigations[110].

Vorinostat is an inhibitor of class I and II HDAC. In combination with BTZ, vorinostat revealed limited results in clinical phase $\mathrm{II}$ and I respectively for relapsing $\mathrm{MCL}$ and $\mathrm{MM}$ [111]. Others combinations involving this HDAC inhibitor are under investigation, and epigenetic regulation mechanisms such as DNA methylation have been subject of preclinical studies. Because NOXA and BCL-2 are demethylated during BTZ treatment, the DNA methytransferase inhibitor decitabine showed synergic effect with BTZ in PI-resistant MCL [112].

\section{Concluding remarks}

Molecular mechanisms that explain inherent or acquired BTZ resistance have been essentially explored in $\mathrm{MM}$ and $\mathrm{MCL}$. The identified alterations include mutations in proteasomal subunits and activation of pro-survival signalling pathways that have impact in cell cycle, cell differentiation, apoptosis, and stabilization of critical cellular factors. Two mayor proteolytic pathways inside the cell, UPS and ALS are major regulators of protein homeostasis and contribute to maintain the balance required to accomplish all protein functions, including the capacity of the cell to respond to BTZ. Beside the malignant cell itself, microenvironment is a crucial factor for impairing PI activity, especially in MM cells, which are heavily dependent on external factors for their growth and response to drugs.

New molecular mechanisms are regularly discovered that have been associated with drug resistance. Some of these mechanisms could be implicated in PI resistance, enhancing the complexity of this process. In the hypothesis of a multifactorial origin, the conception of new and more efficient approaches tackling PI resistance, should include combinatorial approaches simultaneously targeting multiple cellular mechanism. In the case of MCL and $\mathrm{MM}$, the development of new types of drug such as IMiDs and BETis used in combination with PIs led to promising results in vivo. Nevertheless, combinatorial treatment could also increase off-target effects and for this reason a better assessment of these treatments has to be performed before been used in patients. The perfect strategy to overcome drug resistance in $\mathrm{MM}, \mathrm{MCL}$ and other cancer types is far from being identified. Improving our knowledge on the molecular mechanisms implicated in resistance would also open the possibility to elaborate more efficient treatments while reducing the undesired side effects on healthy cells.

\section{Acknowledgments}

MGS and MSR are part of the UbiCODE project and received funding from the European Union's Horizon 2020 research and innovation program under the Marie Skłodowska-Curie grant agreement No 765445. GQ is a fellow from the French Ministry of Education. MSR is also funded by the Institut National du Cancer, France (PLBIO16-251), LASSERLAB-EUROPE grant number 654148 and CONACyT-SRE (Mexico) grant 0280365.

BS acknowledges supports from Ligue contre le Cancer and Fondation Française pour la Recherche contre le Myélome et les Gammapathies. GR was financially supported by 
Fondo de Investigación Sanitaria PI15/00102 and PI18/01383, European Regional Development Fund (ERDF) "Una manera de hacer Europa".

Table 1. Proteasome inhibitors

\begin{tabular}{lllll}
\hline PI & Action & Family & Target & IC $_{50}(\mathbf{n M})$ \\
\hline Bortezomib & Reversible & Boronate & $\beta 5 \mathrm{c}$ & 7 \\
& & & $\beta 5 i$ & 4 \\
& & & $\beta 1 \mathrm{c}$ & 74 \\
\hline Carfilzomib & Irreversible & Epoxyketone & $\beta 5 \mathrm{c}$ & 5 \\
& & & $\beta 5 \mathrm{i}$ & 33 \\
\hline Oprozomib & Irreversible & Epoxyketone & $\beta 5 \mathrm{c}$ & 36 \\
CFZ oral analog & & & $\beta 5 \mathrm{i}$ & 82 \\
\hline Ixazomib & Reversible & Boronate & $\beta 5 \mathrm{c}$ & 3 \\
& & & $\beta 5 i$ & 31 \\
\hline Marizomib & Irreversible & $\beta$-lactone & $\beta 5 \mathrm{c}$ & 2.5 \\
& & & $\beta 2 \mathrm{c}$ & 26 \\
& & & $\beta 1 \mathrm{c}$ & 330 \\
\hline ONX0914 & Irreversible & Epoxyketone & $\beta 5 \mathrm{c}$ & 28 \\
& & & $\beta 5 \mathrm{i}$ & 280 \\
\hline
\end{tabular}

Data presented in the table have been compiled from [4].

Table 2: PSMB5 mutations associated to BTZ resistance in $M C L$ and $M M$

\begin{tabular}{|c|c|c|c|c|c|}
\hline Gene & Mutation & Protein & $\begin{array}{l}\text { Tumour } \\
\text { cells }\end{array}$ & Pathology & Ref. \\
\hline \multirow{5}{*}{ PSMB5 } & c. $322 \mathrm{G}>\mathrm{A}$ & p.A49T & $\begin{array}{l}\text { KMS-11 } \\
\text { OPM-2 }\end{array}$ & $\mathrm{MM}$ & [113] \\
\hline & $\begin{array}{l}\text { c. } 247 A>G \\
\text { c. } 322 G>A\end{array}$ & $\begin{array}{l}\text { p.T21A } \\
\text { p.A49T }\end{array}$ & 8226 & MM & [11] \\
\hline & c. $310 A>G$ & p.M45V & $8226 *$ & MM & {$[4]$} \\
\hline & c. $310 A>G$ & p.M45V & AMO & $\mathrm{MM}$ & [10] \\
\hline & $\begin{array}{l}\text { c. } 235 \mathrm{G}>\mathrm{A} \\
\text { c. } 256 \mathrm{G}>\mathrm{C} \\
\mathrm{c} .312 \mathrm{G}>\mathrm{C} \\
\mathrm{c} .365 \mathrm{G}>\mathrm{A}\end{array}$ & $\begin{array}{l}\text { p.A20T } \\
\text { p.A27P } \\
\text { p.M45I } \\
\text { p.C63Y }\end{array}$ & $\begin{array}{l}\text { Primary } \\
\text { cells }\end{array}$ & MM & [12] \\
\hline
\end{tabular}

The indicated cell lines have been exposed to a continuous pressure of BTZ; except those marked with *, exposed to PR-924, a selective inhibitor of the immunoproteasome. 
Table 3: Drugs currently used to recover sensitivity to BTZ

\begin{tabular}{|c|c|c|c|c|}
\hline Drug Name(s) & mechanism & molecular target & pathology & ref \\
\hline P5091 & Ubiquitin signal & USP7 & MM & [74] \\
\hline b-AP15 & Ubiquitin signal & USP14 and UCHL5 & MM MCL & {$[75,76]$} \\
\hline SJB3-019A & Ubiquitin signal & USP1 & $\mathrm{MM}$ & [77] \\
\hline O-phenanthroline & Ubiquitin signal & RPN11 & MM & [78] \\
\hline Capzimin & Ubiquitin signal & RPN11 & MM & [78] \\
\hline WP1130 & Ubiquitin signal & USP9X, USP5 & $\mathrm{MM}$ & [79] \\
\hline EOAI3402143 & Ubiquitin signal & USP9X and USP24 & MM & [79] \\
\hline VLX1570 & Ubiquitin signal & USP14 & MM & [80] \\
\hline Verapamil & drug transport & $\mathrm{ABCB} 1$ & $\mathrm{MM}$ & [9] \\
\hline Reserpine & drug transport & ABCB1 & MM & [9] \\
\hline Nelfinavir & drug transport & ABCB1 & MM & {$[41,81]$} \\
\hline Lopinavir & drug transport & $\mathrm{ABCB} 1$ & MM & [41] \\
\hline Bafilomycin A & Autophagy & $\begin{array}{l}\text { Vacuolar } \\
\text { ATPase } \\
\text { ATP6V1A }\end{array}$ & MM, MCL & {$[37,82]$} \\
\hline Chloroquine & Autophagy & Lysosome & $\mathrm{MCL}$ & [37] \\
\hline Verteporfin & Autophagy & p62 & $\mathrm{MCL}$ & [37] \\
\hline Orlistat & Autophagy & fatty acid synthase & $\mathrm{MCL}$ & [83] \\
\hline Perifosine & mTOR/ Akt & Akt, PI3K & MM & [87] \\
\hline Dactolisib NVP-BEZ235 & mTOR/ Akt & PI3K, mTOR & $\mathrm{MCL}$ & [84] \\
\hline $\begin{array}{l}\text { Temsirolimus } \\
\text { (Torisel) }\end{array}$ & mTOR/ Akt & mTOR & $M C L$ & [85] \\
\hline $\begin{array}{c}\text { Deforolimus } \\
\text { (Ridaforolimus) }\end{array}$ & mTOR/ Akt & mTOR & MCL & [86] \\
\hline Perillyl alcohol & NF-kB & TG2 signalling & MM MCL & [90] \\
\hline Selinexor & NF-kB & XPO1 & $\mathrm{MM}$ & [89] \\
\hline $\begin{array}{l}\text { Ibrutinib } \\
\text { (Imbruvica) }\end{array}$ & NF-kB & BTK & MM MCL & {$[92,94,95]$} \\
\hline Degrasyn & NF-kB & STAT, DUBs & $\mathrm{MCL}$ & [91] \\
\hline Obatoclax & Bcl-2 proteins & NOXA & $M C L$ & {$[14,96]$} \\
\hline CAMP & Bcl-2 proteins & Mcl-1 & MM & [97] \\
\hline $\begin{array}{l}\text { Lenalidomide } \\
\text { (Revlimid) }\end{array}$ & IMiD & $\begin{array}{c}\text { CRBN } \\
\text {, TNFSF11, CDH5, } \\
\text { PTGS2 }\end{array}$ & $M C L$ & {$[99,100]$} \\
\hline Pomalidomide & IMiD & $\begin{array}{c}\text { CRBN } \\
\text {, TNF, PTGS2 }\end{array}$ & MM & [101-103] \\
\hline
\end{tabular}




\begin{tabular}{|c|c|c|c|c|}
\hline Dexamethasone & $\begin{array}{c}\text { Glucocorticoid } \\
\text { Immunosuppressant }\end{array}$ & $\begin{array}{c}\text { NR3C1, } \\
\text { NROB1, } \\
\text { ANXA1, } \\
\text { NOS2 }\end{array}$ & MM & $\begin{array}{c}{[101,102,} \\
109]\end{array}$ \\
\hline JQ1 & $\begin{array}{c}\text { BET bromodomain } \\
\text { inhibitors }\end{array}$ & BRD4 & MM & {$[104,105]$} \\
\hline CPI203 & $\begin{array}{c}\text { BET bromodomain } \\
\text { inhibitors }\end{array}$ & BRD4 & MM MCL & {$[72,105]$} \\
\hline $\begin{array}{c}\text { PROTACs } \\
\text { (ARV-825) }\end{array}$ & BET specific target & BRD4 and other BET & MM & {$[106]$} \\
\hline Birabresib (OTX015) & $\begin{array}{c}\text { BET bromodomain } \\
\text { inhibitors }\end{array}$ & BRD2,3,4 & MM & {$[103]$} \\
\hline Ricolinostat (ACY-1215) & epigenetic regulators & HDAC6 & MM MCL & {$[109,110]$} \\
\hline Decitabine & epigenetic regulators & $\begin{array}{c}\text { DNA methyl } \\
\text { transferase }\end{array}$ & MCL & {$[112]$} \\
\hline Vorinostat & epigenetic regulators & HDACl & MM MCL & {$[111]$} \\
\hline
\end{tabular}

Figure 1: Molecular mechanisms associated to BTZ-resistance in acquired and resistant $\mathrm{MCL}$ and MM cell models. The pathways involved are: UPS and ALS degradation systems, UPR response, apoptosis, B-cell differentiation, cell cycle regulation and mutations in the 5 subunit of the proteasome. Arrows indicates up, or downregulation of the different molecular mechanisms when compared with non-pathological conditions. See main text for further details.

\section{References}

1. Budenholzer L, Cheng CL, Li Y, Hochstrasser M (2017) Proteasome Structure and Assembly. J Mol Biol 429:3500-3524. https://doi.org/10.1016/j.jmb.2017.05.027

2. Mata-Cantero L, Lobato-Gil S, Aillet F, et al (2015) The Ubiquitin-Proteasome System (UPS) as a Cancer Drug Target: Emerging Mechanisms and Therapeutics. p225-264 In Stress Response Pathways in Cancer. Ed GT Wondrak, Springer 2015.

3. Roeten MSF, Cloos J, Jansen G (2018) Positioning of proteasome inhibitors in therapy of solid malignancies. Cancer Chemother Pharmacol 81:227-243. https://doi.org/10.1007/s00280-017-3489-0

4. Niewerth D, Jansen G, Assaraf YG, et al (2015) Molecular basis of resistance to proteasome inhibitors in hematological malignancies. Drug Resist Updat Rev Comment Antimicrob Anticancer Chemother 18:18-35. https://doi.org/10.1016/j.drup.2014.12.001 
5. Kumar SK, Rajkumar V, Kyle RA, et al (2017) Multiple myeloma. Nat Rev Dis Primer 3:17046. https://doi.org/10.1038/nrdp.2017.46

6. Pérez-Galán P, Dreyling $M$, Wiestner A (2011) Mantle cell lymphoma: biology, pathogenesis, and the molecular basis of treatment in the genomic era. Blood 117:2638. https://doi.org/10.1182/blood-2010-04-189977

7. Diefenbach CSM, O'Connor OA (2010) Mantle cell lymphoma in relapse: the role of emerging new drugs. Curr Opin Oncol 22:419-423. https://doi.org/10.1097/CCO.0b013e32833d58f2

8. Yong K, Gonzalez-McQuire S, Szabo Z, et al (2018) The start of a new wave: Developments in proteasome inhibition in multiple myeloma. Eur J Haematol. 101:220-236. https://doi.org/10.1111/ejh.13071

9. Robak P, Drozdz I, Szemraj J, Robak T (2018) Drug resistance in multiple myeloma. Cancer Treat Rev 70:199-208. https://doi.org/10.1016/j.ctrv.2018.09.001

10. Soriano GP, Besse L, Li N, et al (2016) Proteasome inhibitor-adapted myeloma cells are largely independent from proteasome activity and show complex proteomic changes, in particular in redox and energy metabolism. Leukemia 30:2198-2207. https://doi.org/10.1038/leu.2016.102

11. Franke NE, Niewerth D, Assaraf YG, et al (2012) Impaired bortezomib binding to mutant $\beta 5$ subunit of the proteasome is the underlying basis for bortezomib resistance in leukemia cells. Leukemia 26:757-768. https://doi.org/10.1038/leu.2011.256

12. Barrio S, Stühmer T, Da-Viá M, et al (2018) Spectrum and functional validation of PSMB5 mutations in multiple myeloma. Leukemia. https://doi.org/10.1038/s41375018-0216-8

13. Valentin R, Grabow S, Davids MS (2018) The rise of apoptosis: targeting apoptosis in hematologic malignancies. Blood 132:1248-1264. https://doi.org/10.1182/blood2018-02-791350

14. Pérez-Galán P, Roué G, Villamor N, et al (2007) The BH3-mimetic GX15-070 synergizes with bortezomib in mantle cell lymphoma by enhancing Noxa-mediated activation of Bak. Blood 109:4441-4449. https://doi.org/10.1182/blood-2006-07-034173

15. Kotschy A, Szlavik Z, Murray J, et al (2016) The MCL1 inhibitor S63845 is tolerable and effective in diverse cancer models. Nature 538:477-482. https://doi.org/10.1038/nature19830

16. Morales AA, Kurtoglu M, Matulis SM, et al (2011) Distribution of Bim determines Mcl-

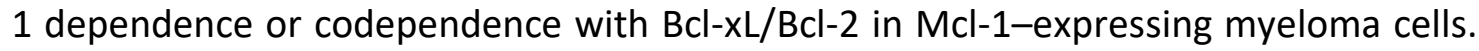
Blood 118:1329-1339. https://doi.org/10.1182/blood-2011-01-327197 
17. Gupta VA, Matulis SM, Conage-Pough JE, et al (2017) Bone marrow microenvironment-derived signals induce $\mathrm{Mcl}-1$ dependence in multiple myeloma. Blood 129:1969-1979. https://doi.org/10.1182/blood-2016-10-745059

18. Pan R, Ruvolo VR, Wei J, et al (2015) Inhibition of Mcl-1 with the pan-Bcl-2 family inhibitor (-)BI97D6 overcomes ABT-737 resistance in acute myeloid leukemia. Blood 126:363-372. https://doi.org/10.1182/blood-2014-10-604975

19. Yang DT, Young KH, Kahl BS, et al (2008) Prevalence of bortezomib-resistant constitutive NF-kappaB activity in mantle cell lymphoma. Mol Cancer 7:1-14. https://doi.org/10.1186/1476-4598-7-40

20. Markovina S, Callander NS, O'Connor SL, et al (2008) Bortezomib-resistant nuclear factor-kappaB activity in multiple myeloma cells. Mol Cancer Res 6:1356-1364. https://doi.org/10.1158/1541-7786.MCR-08-0108

21. Annunziata CM, Davis RE, Demchenko $Y$, et al (2007) Frequent engagement of the classical and alternative NF-kappaB pathways by diverse genetic abnormalities in multiple myeloma. Cancer Cell 12:115-130. https://doi.org/10.1016/j.ccr.2007.07.004

22. Rizzatti EG, Mora-Jensen H, Weniger MA, et al (2008) Noxa mediates bortezomib induced apoptosis in both sensitive and intrinsically resistant mantle cell lymphoma cells and this effect is independent of constitutive activity of the AKT and NF-kappaB $\begin{array}{lll}\text { pathways. Leuk 49:798-808. } & \end{array}$ https://doi.org/10.1080/10428190801910912

23. Manni S, Brancalion A, Mandato E, et al (2013) Protein Kinase CK2 Inhibition Down Modulates the NF-KB and STAT3 Survival Pathways, Enhances the Cellular Proteotoxic Stress and Synergistically Boosts the Cytotoxic Effect of Bortezomib on Multiple Myeloma and Mantle Cell Lymphoma Cells. Clin. Cancer Res 18:1888-1900. https://doi.org/10.1371/journal.pone.0075280

24. Chattopadhyay S, Thomsen H, da Silva Filho MI, et al (2018) Enrichment of B cell receptor signaling and epidermal growth factor receptor pathways in monoclonal gammopathy of undetermined significance: a genome-wide genetic interaction study. Mol Med 24:30. https://doi.org/10.1186/s10020-018-0031-8

25. Kim A, Seong KM, Kang HJ, et al (2015) Inhibition of Lyn is a promising treatment for mantle cell lymphoma with bortezomib resistance. Oncotarget 6:38225-38238

26. Pérez-Galán $\mathrm{P}$, Roué $\mathrm{G}$, Villamor $\mathrm{N}$, et al (2006) The proteasome inhibitor bortezomib induces apoptosis in mantle-cell lymphoma through generation of ROS and Noxa activation independent of p53 status. Blood 107:257-264. https://doi.org/10.1182/blood-2005-05-2091

27. Weniger MA, Rizzatti EG, Pérez-Galán P, et al (2011) Treatment-induced oxidative stress and cellular antioxidant capacity determine response to bortezomib in mantle cell lymphoma. Clin Cancer Res 17:5101-5112. https://doi.org/10.1158/10780432.CCR-10-3367 
28. Luanpitpong S, Poohadsuan J, Samart P, et al (2018) Reactive oxygen species mediate cancer stem-like cells and determine bortezomib sensitivity via Mcl-1 and Zeb-1 in mantle cell lymphoma. Biochim Biophys Acta Mol Basis Dis 1864:3739-3753. https://doi.org/10.1016/j.bbadis.2018.09.010

29. Sánchez-Tilló E, Fanlo L, Siles L, et al (2014) The EMT activator ZEB1 promotes tumor growth and determines differential response to chemotherapy in mantle cell lymphoma. Cell Death Differ 21:247-257. https://doi.org/10.1038/cdd.2013.123

30. Balsas P, Galán-Malo P, Marzo I, Naval J (2012) Bortezomib resistance in a myeloma cell line is associated to PSM 35 overexpression and polyploidy. Leuk Res 36:212-218. https://doi.org/10.1016/j.leukres.2011.09.011

31. Rückrich T, Kraus M, Gogel J, et al (2009) Characterization of the ubiquitin-proteasome system in bortezomib-adapted cells. Leukemia 23:1098-1105. https://doi.org/10.1038/leu.2009.8

32. Shuqing L, Jianmin $\mathrm{Y}$, Chongmei $\mathrm{H}$, et al (2011) Upregulated expression of the PSMB5 gene may contribute to drug resistance in patient with multiple myeloma when treated with bortezomib-based regimen. Exp Hematol 39:1117-1118. https://doi.org/10.1016/j.exphem.2011.09.003

33. Acosta-Alvear D, Cho MY, Wild T, et al (2015) Paradoxical resistance of multiple myeloma to proteasome inhibitors by decreased levels of 195 proteasomal subunits. eLife 4:e08153. https://doi.org/10.7554/eLife.08153

34. Shi C-X, Kortüm KM, Zhu YX, et al (2017) CRISPR Genome-Wide Screening Identifies Dependence on the Proteasome Subunit PSMC6 for Bortezomib Sensitivity in Multiple Myeloma. Mol Cancer Ther 16:2862-2870. https://doi.org/10.1158/1535-7163.MCT$17-0130$

35. Bartel B (2015) Proteaphagy-Selective Autophagy of Inactive Proteasomes. Mol Cell 58:970-971. https://doi.org/10.1016/j.molcel.2015.06.004

36. Marshall RS, Vierstra RD (2018) Autophagy: The Master of Bulk and Selective Recycling. Annu Rev Plant Biol 69:173-208. https://doi.org/10.1146/annurev-arplant042817-040606

37. Quinet G, Rodriguez MS Evidences for an active proteaphagy in Bortezomib Resistant Mantle cell lymphoma cells. Manuscr. Prep. 2019

38. Bond MR, Hanover JA (2013) O-GlcNAc cycling: a link between metabolism and chronic disease. Annu Rev Nutr 33:205-229. https://doi.org/10.1146/annurev-nutr071812-161240

39. Luanpitpong S, Chanthra N, Janan M, et al (2018) Inhibition of O-GlcNAcase Sensitizes Apoptosis and Reverses Bortezomib Resistance in Mantle Cell Lymphoma through Modification of Truncated Bid. Mol Cancer Ther 17:484-496. https://doi.org/10.1158/1535-7163.MCT-17-0390 
40. Abraham I, Jain S, Wu C-P, et al (2010) Marine sponge-derived sipholane triterpenoids reverse P-glycoprotein ( $A B C B 1)$-mediated multidrug resistance in cancer cells. Biochem Pharmacol 80:1497-1506. https://doi.org/10.1016/j.bcp.2010.08.001

41. Besse A, Stolze SC, Rasche L, et al (2018) Carfilzomib resistance due to ABCB1/MDR1 overexpression is overcome by nelfinavir and lopinavir in multiple myeloma. Leukemia 32:391-401. https://doi.org/10.1038/leu.2017.212

42. Fok JHL, Hedayat S, Zhang L, et al (2018) HSF1 Is Essential for Myeloma Cell Survival and A Promising Therapeutic Target. Clin Cancer Res 24:2395-2407. https://doi.org/10.1158/1078-0432.CCR-17-1594

43. Dytfeld D, Luczak M, Wrobel T, et al (2016) Comparative proteomic profiling of refractory/relapsed multiple myeloma reveals biomarkers involved in resistance to bortezomib-based therapy. Oncotarget 7:56726-56736. https://doi.org/10.18632/oncotarget.11059

44. Hayes JD, Dinkova-Kostova AT (2014) The Nrf2 regulatory network provides an interface between redox and intermediary metabolism. Trends Biochem Sci 39:199218. https://doi.org/10.1016/j.tibs.2014.02.002

45. Zong Z-H, Du Z-X, Zhang H-Y, et al (2015) Involvement of Nrf2 in proteasome inhibition-mediated induction of ORP150 in thyroid cancer cells. Oncotarget 7:34163426. https://doi.org/10.18632/oncotarget.6636

46. Riz I, Hawley TS, Marsal JW, Hawley RG (2016) Noncanonical SQSTM1/p62-Nrf2 pathway activation mediates proteasome inhibitor resistance in multiple myeloma cells via redox, metabolic and translational reprogramming. Oncotarget 7:6636066385. https://doi.org/10.18632/oncotarget.11960

47. Starheim KK, Holien T, Misund K, et al (2016) Intracellular glutathione determines bortezomib cytotoxicity in multiple myeloma cells. Blood Cancer J 6:e446. https://doi.org/10.1038/bcj.2016.56

48. Podar K, Chauhan D, Anderson KC (2009) Bone marrow microenvironment and the identification of new targets for myeloma therapy. Leukemia 23:10-24. https://doi.org/10.1038/leu.2008.259

49. Finlayson AE, Freeman KW (2009) A Cell Motility Screen Reveals Role for MARCKSRelated Protein in Adherens Junction Formation and Tumorigenesis. PLOS ONE 4:e7833. https://doi.org/10.1371/journal.pone.0007833

50. Yang Y, Chen Y, Saha MN, et al (2015) Targeting phospho-MARCKS overcomes drugresistance and induces antitumor activity in preclinical models of multiple myeloma. Leukemia 29:715-726. https://doi.org/10.1038/leu.2014.255

51. Sprynski AC, Hose D, Caillot L, et al (2009) The role of IGF-1 as a major growth factor for myeloma cell lines and the prognostic relevance of the expression of its receptor. Blood 113:4614-4626. https://doi.org/10.1182/blood-2008-07-170464 
52. Kuhn DJ, Berkova Z, Jones RJ, et al (2012) Targeting the insulin-like growth factor-1 receptor to overcome bortezomib resistance in preclinical models of multiple myeloma. Blood 120:3260-3270. https://doi.org/10.1182/blood-2011-10-386789

53. Vincenz L, Jäger R, O'Dwyer M, Samali A (2013) Endoplasmic reticulum stress and the unfolded protein response: targeting the Achilles heel of multiple myeloma. Mol Cancer Ther 12:831-843. https://doi.org/10.1158/1535-7163.MCT-12-0782

54. Leung-Hagesteijn C, Erdmann N, Cheung G, et al (2013) Xbp1s-Negative Tumor B Cells and Pre-Plasmablasts Mediate Therapeutic Proteasome Inhibitor Resistance in Multiple Myeloma. Cancer Cell 24:289-304. https://doi.org/10.1016/j.ccr.2013.08.009

55. Mimura N, Fulciniti M, Gorgun G, et al (2012) Blockade of XBP1 splicing by inhibition of IRE1 $\alpha$ is a promising therapeutic option in multiple myeloma. Blood 119:57725781. https://doi.org/10.1182/blood-2011-07-366633

56. Wang C, Pan Y-H, Shan M, et al (2015) Knockdown of UbcH10 enhances the chemosensitivity of dual drug resistant breast cancer cells to epirubicin and docetaxel. Int J Mol Sci 16:4698-4712. https://doi.org/10.3390/ijms16034698

57. Lilienbaum A (2013) Relationship between the proteasomal system and autophagy. Int J Biochem Mol Biol 4:1-26

58. White E, DiPaola RS (2009) The double-edged sword of autophagy modulation in cancer. Clin Cancer Res 15:5308-5316. https://doi.org/10.1158/1078-0432.CCR-075023

59. Ding W-X, Ni H-M, Gao W, et al (2007) Linking of Autophagy to Ubiquitin-Proteasome System Is Important for the Regulation of Endoplasmic Reticulum Stress and Cell Viability. Am J Pathol 171:513-524. https://doi.org/10.2353/ajpath.2007.070188

60. Amaravadi RK, Lippincott-Schwartz J, Yin X-M, et al (2011) Principles and Current Strategies for Targeting Autophagy for Cancer Treatment. Clin Cancer Res 17:654-666. https://doi.org/10.1158/1078-0432.CCR-10-2634

61. Cohen-Kaplan V, Livneh I, Avni N, et al (2016) The ubiquitin-proteasome system and autophagy: Coordinated and independent activities. Int J Biochem Cell Biol 79:403418. https://doi.org/10.1016/j.biocel.2016.07.019

62. Chen S, Zhang Y, Zhou L, et al (2014) A Bim-targeting strategy overcomes adaptive bortezomib resistance in myeloma through a novel link between autophagy and apoptosis. Blood 124:2687-2697. https://doi.org/10.1182/blood-2014-03-564534

63. Wang $\mathrm{Q}$, Mora-Jensen $\mathrm{H}$, Weniger MA, et al (2009) ERAD inhibitors integrate ER stress with an epigenetic mechanism to activate BH3-only protein NOXA in cancer cells. Proc Natl Acad Sci USA 106:2200-2205. https://doi.org/10.1073/pnas.0807611106

64. Ni M, Lee AS (2007) ER chaperones in mammalian development and human diseases. FEBS Lett 581:3641-3651. https://doi.org/10.1016/j.febslet.2007.04.045 
65. Kaufman RJ (2002) Orchestrating the unfolded protein response in health and disease. J Clin Invest 110:1389-1398. https://doi.org/10.1172/JCI16886

66. Szegezdi E, Logue SE, Gorman AM, Samali A (2006) Mediators of endoplasmic reticulum stress-induced apoptosis. EMBO Rep 7:880-885. https://doi.org/10.1038/sj.embor.7400779

67. Roué G, Pérez-Galán P, Mozos A, et al (2011) The Hsp90 inhibitor IPI-504 overcomes bortezomib resistance in mantle cell lymphoma in vitro and in vivo by downregulation of the prosurvival ER chaperone BiP/Grp78. Blood 117:1270-1279. https://doi.org/10.1182/blood-2010-04-278853

68. Weinkauf M, Zimmermann Y, Hartmann E, et al (2009) 2-D PAGE-based comparison of proteasome inhibitor bortezomib in sensitive and resistant mantle cell lymphoma. Electrophoresis 30:974-986. https://doi.org/10.1002/elps.200800508

69. Davenport EL, Moore HE, Dunlop AS, et al (2007) Heat shock protein inhibition is associated with activation of the unfolded protein response pathway in myeloma plasma cells. Blood 110:2641-2649. https://doi.org/10.1182/blood-2006-11-053728

70. Roué $\mathrm{G}$, Pichereau V, Lincet $\mathrm{H}$, et al (2008) Cyclin D1 mediates resistance to apoptosis through upregulation of molecular chaperones and consequent redistribution of cell death regulators. Oncogene 27:. https://doi.org/10.1038/onc.2008.126

71. Orchard J, Garand R, Davis Z, et al (2003) A subset of $t(11 ; 14)$ lymphoma with mantle cell features displays mutated IgVH genes and includes patients with good prognosis, nonnodal disease. Blood 101:4975-4981. https://doi.org/10.1182/blood-2002-061864

72. Moros A, Rodríguez V, Saborit-Villarroya I, et al (2014) Synergistic antitumor activity of lenalidomide with the BET bromodomain inhibitor CPI203 in bortezomib-resistant mantle cell lymphoma. Leukemia 28:2049-2059. https://doi.org/10.1038/leu.2014.106

73. Desai S, Maurin M, Smith MA, et al (2010) PRDM1 is required for Mantle Cell Lymphoma response to Bortezomib. Mol Cancer Res MCR 8:907-918. https://doi.org/10.1158/1541-7786.MCR-10-0131

74. Chauhan D, Tian Z, Nicholson B, et al (2012) A Small Molecule Inhibitor of UbiquitinSpecific Protease-7 Induces Apoptosis in Multiple Myeloma Cells and Overcomes Bortezomib Resistance. Cancer Cell 22:345-358. https://doi.org/10.1016/j.ccr.2012.08.007

75. Tian Z, D'Arcy P, Wang X, et al (2014) A novel small molecule inhibitor of deubiquitylating enzyme USP14 and UCHL5 induces apoptosis in multiple myeloma and overcomes bortezomib resistance. Blood 123:706-716. https://doi.org/10.1182/blood-2013-05-500033

76. Kropp KN, Maurer S, Rothfelder K, et al (2018) The novel deubiquitinase inhibitor bAP15 induces direct and NK cell-mediated antitumor effects in human mantle cell 
lymphoma. Cancer Immunol Immunother 67:935-947. https://doi.org/10.1007/s00262-018-2151-y

77. Das DS, Das A, Ray A, et al (2017) Blockade of Deubiquitylating Enzyme USP1 Inhibits DNA Repair and Triggers Apoptosis in Multiple Myeloma Cells. Clin Cancer Res 23:4280-4289. https://doi.org/10.1158/1078-0432.CCR-16-2692

78. Song Y, Li S, Ray A, et al (2017) Blockade of deubiquitylating enzyme Rpn11 triggers apoptosis in multiple myeloma cells and overcomes bortezomib resistance. Oncogene 36:5631-5638. https://doi.org/10.1038/onc.2017.172

79. Peterson LF, Sun H, Liu Y, et al (2015) Targeting deubiquitinase activity with a novel small-molecule inhibitor as therapy for B-cell malignancies. Blood 125:3588-3597. https://doi.org/10.1182/blood-2014-10-605584

80. Wang X, Mazurkiewicz M, Hillert EK, et al (2016) The proteasome deubiquitinase inhibitor VLX1570 shows selectivity for ubiquitin-specific protease-14 and induces apoptosis of multiple myeloma cells | Scientific Reports 6:26979. https://doi.org/10.1038/srep26979

81. Driessen C, Kraus M, Joerger M, et al (2016) Treatment with the HIV protease inhibitor nelfinavir triggers the unfolded protein response and may overcome proteasome inhibitor resistance of multiple myeloma in combination with bortezomib: a phase I trial (SAKK 65/08). Haematologica 101:346-355. https://doi.org/10.3324/haematol.2015.135780

82. Kawaguchi T, Miyazawa K, Moriya S, et al (2011) Combined treatment with bortezomib plus bafilomycin A1 enhances the cytocidal effect and induces endoplasmic reticulum stress in U266 myeloma cells: crosstalk among proteasome, autophagy-lysosome and ER stress. Int J Oncol 38:643-654. https://doi.org/10.3892/ijo.2010.882

83. Heine S, Kleih M, Giménez N, et al (2018) Cyclin D1-CDK4 activity drives sensitivity to bortezomib in mantle cell lymphoma by blocking autophagy-mediated proteolysis of NOXA. J Hematol Oncol 11:112. https://doi.org/10.1186/s13045-018-0657-6

84. Kim A, Park S, Lee J-E, et al (2012) The dual PI3K and mTOR inhibitor NVP-BEZ235 exhibits anti-proliferative activity and overcomes bortezomib resistance in mantle cell lymphoma cells. Leuk Res 36:912-920. https://doi.org/10.1016/j.leukres.2012.02.010

85. Witzig TE, Geyer SM, Ghobrial I, et al (2005) Phase II trial of single-agent temsirolimus (CCl-779) for relapsed mantle cell lymphoma. J Clin Oncol 23:5347-5356. https://doi.org/10.1200/JCO.2005.13.466

86. Rizzieri DA, Feldman E, Dipersio JF, et al (2008) A phase 2 clinical trial of deforolimus (AP23573, MK-8669), a novel mammalian target of rapamycin inhibitor, in patients with relapsed or refractory hematologic malignancies. Clin Cancer Res 14:2756-2762. https://doi.org/10.1158/1078-0432.CCR-07-1372 
87. Richardson PG, Eng C, Kolesar J, et al (2012) Perifosine, an oral, anti-cancer agent and inhibitor of the Akt pathway: mechanistic actions, pharmacodynamics, pharmacokinetics, and clinical activity. Expert Opin Drug Metab Toxicol 8:623-633. https://doi.org/10.1517/17425255.2012.681376

88. Turner JG, Kashyap T, Dawson JL, et al (2016) XPO1 inhibitor combination therapy with bortezomib or carfilzomib induces nuclear localization of $1 \mathrm{kB} \alpha$ and overcomes acquired proteasome inhibitor resistance in human multiple myeloma. Oncotarget 7:78896-78909. https://doi.org/10.18632/oncotarget.12969

89. Muz B, Azab F, de la Puente P, et al (2017) Selinexor Overcomes Hypoxia-Induced Drug Resistance in Multiple Myeloma. Transl Oncol 10:632-640. https://doi.org/10.1016/j.tranon.2017.04.010

90. Jung HJ, Chen Z, Wang M, et al (2012) Calcium blockers decrease the bortezomib resistance in mantle cell lymphoma via manipulation of tissue transglutaminase activities. Blood 119:2568-2578. https://doi.org/10.1182/blood-2011-09-377598

91. Pham LV, Tamayo AT, Li C, et al (2010) Degrasyn potentiates the antitumor effects of bortezomib in Mantle cell lymphoma cells in vitro and in vivo: therapeutic implications. Mol Cancer Ther 9:2026-2036. https://doi.org/10.1158/1535-7163.MCT10-0238

92. de Claro RA, McGinn KM, Verdun N, et al (2015) FDA Approval: Ibrutinib for Patients with Previously Treated Mantle Cell Lymphoma and Previously Treated Chronic Lymphocytic Leukemia. Clin Cancer Res 21:3586-3590. https://doi.org/10.1158/10780432.CCR-14-2225

93. Campo E, Rule S (2015) Mantle cell lymphoma: evolving management strategies. Blood 125:48-55. https://doi.org/10.1182/blood-2014-05-521898

94. Murray MY, Zaitseva L, Auger MJ, et al (2015) Ibrutinib inhibits BTK-driven NF-KB p65 activity to overcome bortezomib-resistance in multiple myeloma. Cell Cycle 14:23672375. https://doi.org/10.1080/15384101.2014.998067

95. Richardson PG, Bensinger WI, Huff CA, et al (2018) Ibrutinib alone or with dexamethasone for relapsed or relapsed and refractory multiple myeloma: phase 2 trial results. Br J Haematol 180:821-830. https://doi.org/10.1111/bjh.15058

96. Goy A, Hernandez-IIzaliturri FJ, Kahl B, et al (2014) A phase I/II study of the pan Bcl-2 inhibitor obatoclax mesylate plus bortezomib for relapsed or refractory mantle cell lymphoma. Leuk Lymphoma 55:2761-2768. https://doi.org/10.3109/10428194.2014.907891

97. Wang $\mathrm{Y}$, Tang $\mathrm{Y}$, Hang $\mathrm{H}$, et al (2018) cAMP induces cell apoptosis in multiple myeloma and overcomes bortezomib resistance. Am J Cancer Res 8:16-29

98. Lopez-Girona A, Heintel D, Zhang L-H, et al (2011) Lenalidomide downregulates the cell survival factor, interferon regulatory factor-4, providing a potential mechanistic 
link for predicting response. $\mathrm{Br} J$ Haematol 154:325-336. https://doi.org/10.1111/j.1365-2141.2011.08689.x

99. Desai M, Newberry K, Ou Z, et al (2014) Lenalidomide in relapsed or refractory mantle cell lymphoma: overview and perspective. Ther Adv Hematol 5:91-101. https://doi.org/10.1177/2040620714532124

100. Morrison VA, Jung S-H, Johnson J, et al (2015) Therapy with bortezomib plus lenalidomide for relapsed/refractory mantle cell lymphoma: final results of a phase II trial (CALGB 50501). Leuk Lymphoma 56:958-964. https://doi.org/10.3109/10428194.2014.938333

101. Richardson PG, Hofmeister CC, Raje NS, et al (2017) Pomalidomide, bortezomib and low-dose dexamethasone in lenalidomide-refractory and proteasome inhibitorexposed myeloma. Leukemia 31:2695-2701. https://doi.org/10.1038/leu.2017.173

102. Spencer A, Harrison S, Zonder J, et al (2018) A phase 1 clinical trial evaluating marizomib, pomalidomide and low-dose dexamethasone in relapsed and refractory multiple myeloma (NPI-0052-107): final study results. $\mathrm{Br} J$ Haematol 180:41-51. https://doi.org/10.1111/bjh.14987

103. Tarantelli C, Bernasconi E, Gaudio E, et al (2018) BET bromodomain inhibitor birabresib in mantle cell lymphoma: in vivo activity and identification of novel combinations to overcome adaptive resistance. ESMO Open 3:e000387. https://doi.org/10.1136/esmoopen-2018-000387

104. Delmore JE, Issa GC, Lemieux ME, et al (2011) BET bromodomain inhibition as a therapeutic strategy to target c-Myc. Cell 146:904-917. https://doi.org/10.1016/j.cell.2011.08.017

105. Siegel MB, Liu SQ, Davare MA, et al (2015) Small molecule inhibitor screen identifies synergistic activity of the bromodomain inhibitor CPI203 and bortezomib in drug resistant myeloma. Oncotarget 6:18921-18932

106. Zhang X, Lee HC, Shirazi F, et al (2018) Protein Targeting Chimeric Molecules Specific for Bromodomain and Extra-terminal Motif Family Proteins are Active Against PreClinical Models of Multiple Myeloma. Leukemia 32:2224-2239. https://doi.org/10.1038/s41375-018-0044-x

107. McConkey DJ, White M, Yan W (2012) HDAC inhibitor modulation of proteotoxicity as a therapeutic approach in cancer. Adv Cancer Res 116:131-163. https://doi.org/10.1016/B978-0-12-394387-3.00004-5

108. Catley L, Weisberg E, Kiziltepe T, et al (2006) Aggresome induction by proteasome inhibitor bortezomib and alpha-tubulin hyperacetylation by tubulin deacetylase (TDAC) inhibitor LBH589 are synergistic in myeloma cells. Blood 108:3441-3449. https://doi.org/10.1182/blood-2006-04-016055 
109. Vog| DT, Raje N, Jagannath S, et al (2017) Ricolinostat, the First Selective Histone Deacetylase 6 Inhibitor, in Combination with Bortezomib and Dexamethasone for Relapsed or Refractory Multiple Myeloma. Clin Cancer Res 23:3307-3315. https://doi.org/10.1158/1078-0432.CCR-16-2526

110. Amengual JE, Johannet $P$, Lombardo $M$, et al (2015) Dual Targeting of Protein Degradation Pathways with the Selective HDAC6 Inhibitor ACY-1215 and Bortezomib Is Synergistic in Lymphoma. Clin Cancer Res 21:4663-4675. https://doi.org/10.1158/1078-0432.CCR-14-3068

111. Yazbeck V, Shafer D, Perkins EB, et al (2018) A Phase II Trial of Bortezomib and Vorinostat in Mantle Cell Lymphoma and Diffuse Large B-cell Lymphoma. Clin Lymphoma Myeloma Leuk 18:569-575.e1. https://doi.org/10.1016/j.clml.2018.05.023

112. Leshchenko VV, Kuo P-Y, Jiang Z, et al (2015) Harnessing Noxa demethylation to overcome Bortezomib resistance in mantle cell lymphoma. Oncotarget 6:2733227342. https://doi.org/10.18632/oncotarget.2903

113. Ri M, lida S, Nakashima T, et al (2010) Bortezomib-resistant myeloma cell lines: a role for mutated PSMB5 in preventing the accumulation of unfolded proteins and fatal ER stress. Leukemia 24:1506-1512. https://doi.org/10.1038/leu.2010.137 


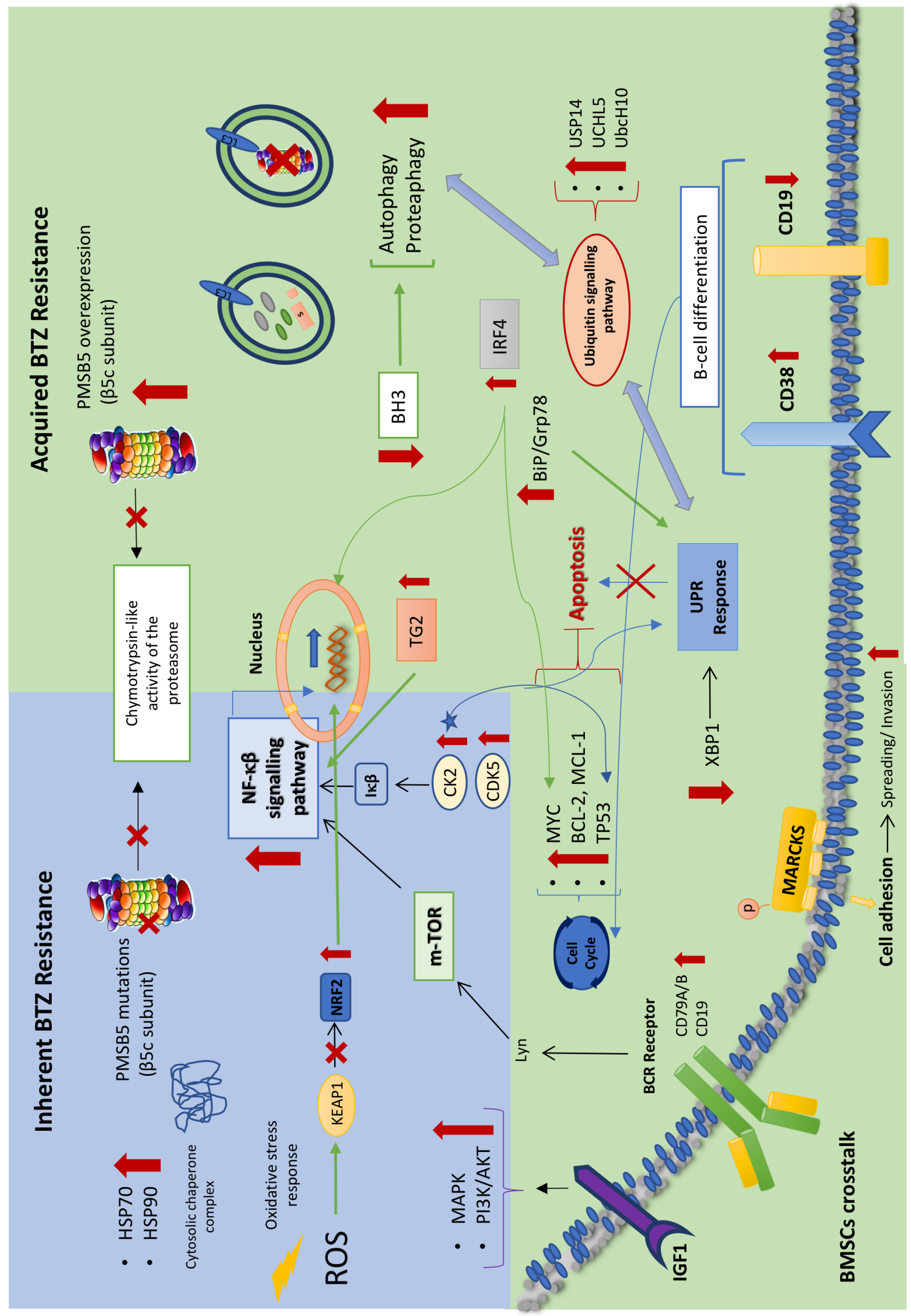

